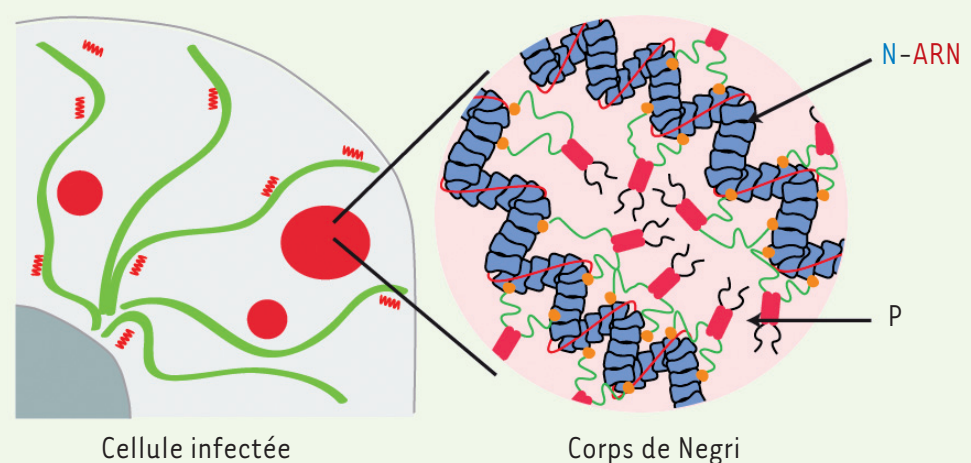

Cellule infectée

Corps de Negri

Figure 2. Schématisation d'un corps de Negri. Dans la cellule infectée, cohabitent quelques gros corps de Negri en rouge (un ou deux par cellule qui se sont formés autour des génomes des virus entrants), des corps de Negri de taille moyenne (formés autour des génomes synthétisés dans la cellule infectée) et des nucléocapsides isolées. Les corps de Negri contiennent essentiellement des nucléocapsides ( $\mathrm{N}-\mathrm{ARN}$ ) et de la phosphoprotéine P. La cohésion de la phase liquide est probablement assurée par des interactions faibles entre les domaines intrinsèquement désordonnés de $P$.

Enfin, la découverte du caractère liquide des usines virales ouvre de nouvelles pistes thérapeutiques. Des drogues capables de disperser la phase liquide les constituant devraient être efficaces contre l'infection. $\diamond$

Rabies virus factories are formed by liquid-liquid phase separation

\section{LIENS D'INTÉRÊT}

Les auteurs déclarent n'avoir aucun lien d'intérêt concernant les données publiées dans cet article.

NOUVELLE

\title{
Un point de contrôle développemental synchronise la morphogenèse et la différenciation cellulaire dans l'embryon de mammifère
}

Julien G. Dumortier, Jean-Léon Maître

> Pendant le développement embryonnaire, la destinée des cellules est déterminée par les informations sur leurs positions dont elles disposent. La différenciation cellulaire déclenche souvent des modifications dans l'organisation de l'embryon qui, en retour, créent de nouvelles informations de positionnement. Ces cycles de morphogénèse et de différenciation sont des processus indis-

\section{RÉFÉRENCES}

1. Nikolic J, Blondel D. Le virus de la rage induit la formation de granules de stress en contact étroit avec les usines virales. Med Sci (Paris) 2017 ; 33 : 921-3.

2. Netherton $\mathrm{CL}$, Wileman T. Virus factories, double membrane vesicles and viroplasm generated in animal cells. Curr Opin Virol $2011 ; 1$ : 381-7.

3. Schramm B, Locker JK. Cytoplasmic organization of POXvirus DNA replication. Traffic $2005 ; 6$ : 839-46.

4. Harak C, Lohmann V. Ultrastructure of the replication sites of positive-strand RNA viruses. Virology 2015 ; 479-480: 418-33.

5. Hoenen T, Shabman RS, Groseth A, et al. Inclusion bodies are a site of ebolavirus replication. J Virol $2012 ; 86$ : 11779-88.

6. Lahaye X, Vidy A, Pomier C, et al. Functional characterization of Negri bodies (NBs) in rabies virusinfected cells: evidence that NBs are sites of viral transcription and replication. J Virol 2009; $83: 7948-58$

7. Rincheval V, Lelek M, Gault $\varepsilon$, et al. Functional organization of cytoplasmic inclusion bodies in cells infected by respiratory syncytial virus. Nat Commun $2017 ; 8: 563$

8. Negri A. Contributo allo studio dell' eziologia della rabia. Bol Soc Med Chir Pavia 1903 ; 2 : 88-114.

9. Ribadeau-Dumas F, Dacheux L, Bourhy H. La rage. Med Sci (Paris) $2013 ; 29: 47-55$.

10. Nikolic J, Le Bars R, Lama Z, et al. Negri bodies are viral factories with properties of liquid organelles. Nat Commun $2017 ; 8: 58$.

Unité génétique et biologie du développement, Institut Curie, PSL Research University, CNRS UMR3215, Inserm U934, 75248 Paris, France. jean-leon.maitre@curie.fr

pas atteints. Une étude de Shahbazi et ses collègues, parue récemment dans Nature [1], révèle les mécanismes d'un point de contrôle développemental qui agit durant les tout premiers jours de l'embryon du mammifère.

Chez de nombreux mammifères, aux stades initiaux du développement, l'embryon forme une structure, appelée blastocyste, qui s'implante dans l'utérus 


\section{État « naï »}

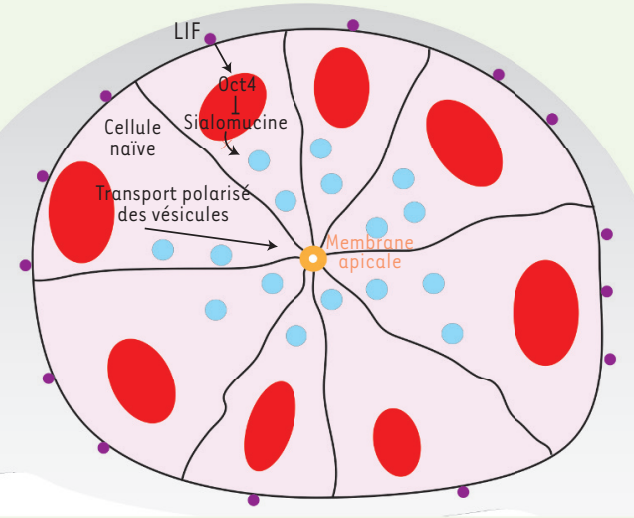

État « amorcé »

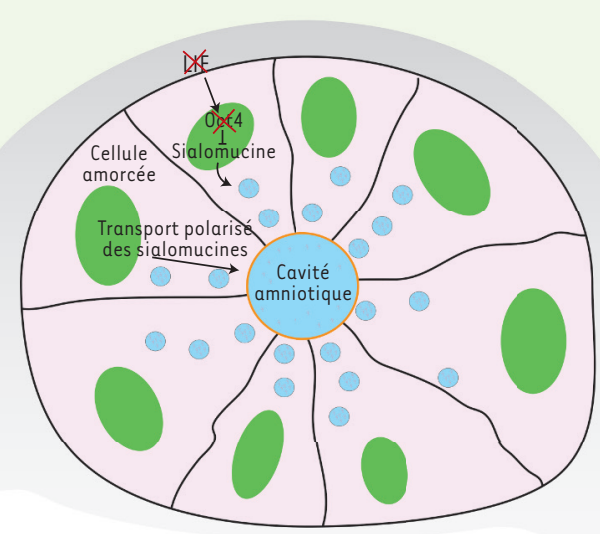

Figure 1. Lorsque l'embryon humain ou murin s'implante dans l'utérus, la cavité amniotique, dans laquelle le fœtus se développe, se forme au sein de l'épiblaste (en rose). Les cellules de l'épiblaste rassemblent leurs bords apicaux (en orange) et orientent leur trafic vésiculaire vers le point de rencontre. Tant que la protéine LIF (leukaemia inhibitory factor), produite par le tissu extra-embryonnaire (en gris), est présente, aucune cavité ne se forme. Simultanément, le LIF maintient l'épiblaste à l'état pluripotent pendant lequel s'exprime le facteur de transcription 0ct4 (en rouge). Quand le LIF cesse d'être produit, les cellules de l'épiblaste initient leur différenciation (en vert), ne produisent plus d'Oct4 et commencent à exprimer des sialomucines. Ces sialomucines, chargées négativement, permettent aux membranes apicales de se repousser mutuellement et rendent possible l'ouverture de la cavité amniotique.

maternel [2]. Cette implantation peut être mise en suspend par l'action d'hormones maternelles qui induisent un état de dormance du blastocyste appelé diapause [3]. Cette diapause est supposée avoir eu un impact important dans l'évolution et dans l'apparition d'un état «naïf » des cellules souches embryonnaires (cellules ES) [3]. En effet, les cellules pluripotentes de l'embryon, mises hormones maternelles qui contrôlent la diapause, et maintient la pluripotence des cellules.

En sortie de diapause, les cellules doivent non seulement initier leur programme de différenciation, mais également redémarrer le programme morphogénétique incluant la formation de la cavité amniotique, une cavité qui va accueillir l'embryon et les fluides qui l'entourent lors de la gestation.

La formation de cavités aqueuses est commune dans le développement embryonnaire (par exemple, les néphrons dans le rein, les vaisseaux sanguins ou les ventricules cérébraux). Les événements moléculaires et cellulaires conduisant à la formation de telles cavités ont été caractérisés in vitro, notamment en utilisant des lignées de cellules épithéliales, qui maintiennent un contact intercellulaire fort, et une polarité apico-basale [5]. Dans certaines conditions, les cellules épithéliales forment une lumière au niveau de leur pôle apical, via un transport polarisé de fluide et de protéines. Ces protéines sont de type sialomucine, des protéines présentant des acides sialiques et chargées négativement. Elles permettront de repousser, électriquement, les membranes apicales. Les protéines de jonctions serrées empêcheront le fluide de s'échapper en réalisant des contacts entre cellules, assurant ainsi l'imperméabilité de la cavité.

Le maintien des cellules dans un état naïf jouerait donc un rôle de point de contrôle dans le développement embryonnaire. II éviterait la formation prématurée de la cavité amniotique. Ce rôle reste à démontrer in vivo. En mettant à profit les méthodes de culture d'embryons ex utero $[6,7]$, Shahbazi et ses collègues ont révélé le mécanisme coordonnant la fin de la pluripotence et la formation de la cavité amniotique.

Ces auteurs ont ainsi, d'abord, noté que la baisse d'expression des gènes de la pluripotence coïncidait avec l'apparition de la cavité amniotique, à la fois chez l'homme et chez la souris. Ils ont ensuite 
observé que le maintien dans un état naïf, par l'apport artificiel de LIF, empêchait les embryons de former une cavité. Pour disséquer la régulation moléculaire précise qui lierait la sortie de la pluripotence avec la formation de la cavité, les auteurs ont utilisé les cellules ES comme substitut de l'embryon. Ils ont ainsi pu montrer que, dans leur état naïf, les cellules $E S$ se réarrangent en rosette, polarisant leurs interfaces, leurs jonctions serrées et leur transport vésiculaire, mais ne formaient pas de cavité. Les cellules restent donc prêtes, dans cette configuration, jusqu'à ce que l'inhibition par le LIF soit levée. Alors, les cellules ES déclenchent l'expression de sialomucines, dont Podxl (podocalyxin), et forment une cavité. Une étape clé contrôlant la coordination entre formation de la cavité et sortie de la pluripotence est donc l'expression de ces sialomucines, qui permettraient aux membranes apicales de devenir répulsives.

Les auteurs ont ensuite analysé les données génomiques disponibles afin de déterminer comment la fin de la pluripotence peut contrôler la formation de la cavité. Cette analyse a révélé un rôle plausible du facteur de transcription Oct4 dans la régulation de l'expression des sialomucines (Figure I). En considérant une récente étude qui décrit les effets de l'absence de 0ct4 dans l'embryon préimplantatoire humain [8], ces résultats suggèrent plusieurs fonctions clés de ce facteur dans le développement précoce des embryons humains. Bien que l'expression des sialomucines soit nécessaire, elle n'est pas suffisante pour induire la formation de la cavité. Les sialomucines requièrent donc la présence d'autres protéines, comme la cinguline, une protéine de jonctions serrées exprimée en l'absence de LIF. Une étude récente a en effet suggéré que cette protéine permettait l'ancrage de vésicules à la membrane apicale des cellules lors de la formation de cavités [9]. De nouvelles études permettront sans aucun doute d'identifier le cocktail de molécules suffisant pour déclencher la formation de la cavité par les cellules naïves.

Le travail de Shahbazi et de ses collègues a mis en évidence un mécanisme par lequel les embryons coordonnent la fin de la pluripotence avec la formation de la cavité amniotique. Dans ce scénario, les cellules naïves forment un épithélium polarisé et mettent en suspens la cavitation. Ce n'est que lorsque les cellules deviennent «amorcées » que leurs membranes apicales se repoussent, permettant ainsi l'accumulation de liquide. Ceci fournit les premiers éléments d'un rôle biologique de l'état naï, agissant comme un point de contrôle, assurant que la cavité amnio- tique ne se forme que lorsque les cellules se sont engagées dans leurs voies de différenciation. Chez l'embryon, la morphogenèse et la différenciation sont ainsi synchrones. $\diamond$

A developmental checkpoint synchronizes morphogenesis and cellular differentiation in mammalian embryos

\section{LIENS D'INTÉRÊT}

Les auteurs déclarent n'avoir aucun lien d'intérêt concernant les données publiées dans cet article.

\section{RÉFÉRENCES}

1. Shahbazi MN, Scialdone A, Skorupska N, et al. Pluripotent state transitions coordinate morphogenesis in mouse and human embryos. Nature $2017 ; 552$ : 239-43.

2. Maître JL. Mechanics of blastocyst morphogenesis. Biol Cell 2017 ; 109 : 323-38.

3. Frankenberg SR, Barros FRO de, Rossant J, et al. The mammalian blastocyst. Wiley Interdiscip Rev Dev Biol 2016; $5: 210-32$.

4. Ying (L, Wray J, Nichols J, et al. The ground state of embryonic stem cell self-renewal. Nature 2008 ; 453 : 519-23.

5. Blasky AJ, Mangan A, Prekeris R. Polarized protein transport and lumen formation during epithelial tissue morphogenesis. Annu Rev Cell Dev Biol 2015 ; 31 : 575-91.

6. Shahbazi MN, Jedrusik A, Vuoristo $S$, et al. Selforganization of the human embryo in the absence of maternal tissues. Nat Cell Biol 2016 ; 18 : 700-8.

7. Bedzhov I, Zernicka-Goetz M. Self-organizing properties of mouse pluripotent cells initiate morphogenesis upon implantation. Cell $2014 ; 156$ : 1032-44.

8. Fogarty NME, MCCarthy A, Snijders KE, et al. Genome editing reveals a role for OCT4 in human embryogenesis. Nature $2017 ; 550: 67-73$.

9. Mangan AJ, Sietsema D V., Li D, et al. Cingulin and actin mediate midbody-dependent apical lumen formation during polarization of epithelial cells. Nat Commun $2016 ; 7: 1-15$.

\section{LA FONDATION PREMUP : UN OPÉRATEUR DE TERRAIN EN PÉRINATALITÉ RECONNU POUR SON EXCELLENCE ET SON INTERDISCIPLINARITÉ}

La Fondation de coopération scientifique PremUp, unique en Europe, intervient sur la prévention du handicap à la naissance, par la protection de la santé de la femme enceinte et du nouveau-né.

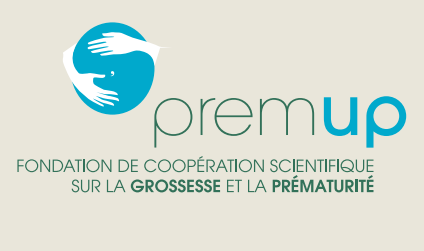

15 UNIVERSTÉ $\$$ PAR DESCARTES

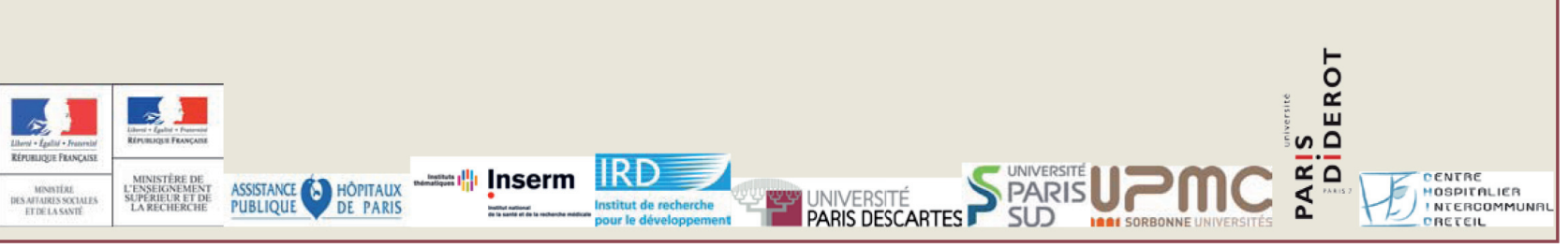

